\title{
5D MODELLING: AN EFFICIENT APPROACH FOR CREATING SPATIOTEMPORAL PREDICTIVE 3D MAPS OF LARGE-SCALE CULTURAL RESOURCES
}

\author{
Anastasios Doulamis ${ }^{\mathrm{a}, *}$, Nikolaos Doulamis $^{\mathrm{a}}$, Charalabos Ioannidis $^{\mathrm{a}}$, Christina Chrysouli $^{\mathrm{b}}$, Nikos Grammalidis $^{\mathrm{b}}$, \\ Kosmas Dimitropoulos ${ }^{\mathrm{b}}$, Chryssy Potsiou ${ }^{\mathrm{a}}$, Elisavet Konstantina Stathopoulou ${ }^{\mathrm{a}}$, Marinos Ioannides ${ }^{\mathrm{c}}$ \\ a National Technical University of Athens, 9 Iroon Polytechniou St., Athens, Greece - \{adoulam@cs.ntua.gr, ndoulam@cs.ntua.gr, \\ cioannid@survey.ntua.gr, chryssy.potsiou@gmail.com $\}$ \\ ${ }^{\mathrm{b}}$ Information Technologies Institute, Centre for Research and Technology Hellas, Thessaloniki, Greece - \{chrysouli, \\ ngramm,dimitrop $\} @$ iti.gr \\ ${ }^{\mathrm{c}}$ Cyprus University of Technology, Limassol, Cyprus - marinos.ioannides@cut.ac.cy
}

KEY WORDS: Large scale Cultural Heritage sites, Cultural Heritage Model, 5D modelling, Selective modelling, CityGML, Visualization

\begin{abstract}
:
Outdoor large-scale cultural sites are mostly sensitive to environmental, natural and human made factors, implying an imminent need for a spatio-temporal assessment to identify regions of potential cultural interest (material degradation, structuring, conservation). On the other hand, in Cultural Heritage research quite different actors are involved (archaeologists, curators, conservators, simple users) each of diverse needs. All these statements advocate that a 5D modelling (3D geometry plus time plus levels of details) is ideally required for preservation and assessment of outdoor large scale cultural sites, which is currently implemented as a simple aggregation of 3D digital models at different time and levels of details. The main bottleneck of such an approach is its complexity, making 5D modelling impossible to be validated in real life conditions. In this paper, a cost effective and affordable framework for 5D modelling is proposed based on a spatial-temporal dependent aggregation of 3D digital models, by incorporating a predictive assessment procedure to indicate which regions (surfaces) of an object should be reconstructed at higher levels of details at next time instances and which at lower ones. In this way, dynamic change history maps are created, indicating spatial probabilities of regions needed further 3D modelling at forthcoming instances. Using these maps, predictive assessment can be made, that is, to localize surfaces within the objects where a high accuracy reconstruction process needs to be activated at the forthcoming time instances. The proposed 5D Digital Cultural Heritage Model (5D-DCHM) is implemented using open interoperable standards based on the CityGML framework, which also allows the description of additional semantic metadata information. Visualization aspects are also supported to allow easy manipulation, interaction and representation of the 5D-DCHM geometry and the respective semantic information. The open source 3DCityDB incorporating a PostgreSQL geo-database is used to manage and manipulate 3D data and their semantics.
\end{abstract}

\footnotetext{
* Corresponding author
} 


\section{INTRODUCTION}

Digitalizing archaeological sites and objects and creating 3D digital models is an important task to preserve Cultural Heritage $(\mathrm{CH})$. Among all $\mathrm{CH}$ resources, the outdoor large-scale archaeological sites are mostly sensitive to weather conditions, natural phenomena (earthquakes, flooding, etc), excavation procedures, and restoration protocols. This implies an imminent need for a spatio-temporal monitoring of those sites to identify regions of potential material degradation, unstable structuring conditions, localize spatial modifications and detect environmental damages (Makantasis et al, 2015). A time varying $3 \mathrm{D}$ model should be developed to assess spatial and temporal diversity of $\mathrm{CH}$ objects but again under a costeffective framework able to be applied to large-scale sites.

On the other hand, several actors are involved in $\mathrm{CH}$ community. $\mathrm{CH}$ researchers and archaeologists can better document sites and objects $(\mathrm{CH}$ resources $)$, relate their operational uses with past social and political structures, economical factors and past advances in science and technology (Gomes, 2014).

Conservators can better assess the effect of different restoration methods on maintaining the structure and the nature of the cultural items, while simultaneously simulate and predict material degradation factors (Ene, 2011).

Curators can exploit the digital counterparts to design exhibitions and disseminate cultural knowledge to wide public (Kitamura, 2013).

Creative industries and Small Medium Enterprises (SME's) can build new services promoting Europe's culture worldwide, forging Europe's cohesion and integration through its diverse culture legacy and boosting European economy. During the severe economic crisis of the last years, creative enterprises and tourism industries are some good examples of withstanding economic recession and (though slightly) provide facts towards sustainable growth of Europe's economy (Bjerke, 2013).

Finally, the general public or site visitors can share unique cultural experiences on handling digital $\mathrm{CH}$ objects and navigating through a digital cultural world.

Each of the aforementioned players presents different needs regarding digital model parameters and levels of details (LoD). Therefore, we need to develop digital models able to respect all diverse requirements of the $\mathrm{CH}$ community users. In addition, the scaled 3D models should be developed under a costeffective way to make them applicable for large scale cultural sites and for different $\mathrm{CH}$ players at a minimal cost.

For instance, for documentation purposes high resolution model is needed; however, to assess the status and the behaviour of a monument regarding its stability in an earthquake, a low resolution model should be accomplished as the computational cost needed to implement finite elements analysis cannot be affordable using a high resolution model. Thus, the need for different levels of details is necessary for such a process.

Currently, 3D digital models are generated under a spatialtemporal independent framework. This means that digitalization information of common parts (surfaces) of an object is not exploited to digitalize similar surfaces of the same or other objects. Furthermore, the digitization process at a current time instance does not exploit results from reconstructions obtained at previous time instances. Last but not least, the LoD dimension of each 3D model is generated differently per each user's category (e.g., $\mathrm{CH}$ researchers, curators, visitors) leading to the creation of independent scaled 3D models. As an additional drawback, one can also refer to the lack of a semantic enrichment of the digital information to assist $\mathrm{CH}$ community users in their research and work.

\subsection{Our Contribution}

In this paper, a scale (LoD) - time varying 3D modelling framework for outdoor large-scale cultural heritage resources is proposed. The proposed framework supports a spatial-temporal dependent approach for cost-effective $3 \mathrm{D}$ reconstruction of $\mathrm{CH}$ objects.

Instead of performing a continuous generation of 3D models, which is a time consuming process, we need (i) to create a high resolution model (first time instance), (ii) to generate low resolution models at next periods (time instances) using simple capturing methods like photos or other sensors, (iii) to match semantic modules of a monument between the high and the low resolution levels, (iv) in areas that there is no salient difference to use the high resolution model to update the current status, (v) in problematic areas to repeat the modelling but only in these limited regions. This procedure will save time and cost.

The model also introduces a framework for semantically enriching the 3D models using interoperable XML schemas like CityGML. This allows easy archiving, manipulation and retrieval, of $3 \mathrm{D}$ captured heritage objects in geospatial databases. Using the metadata structure, we can enhance edocumentation of the content. The whole framework permits the creation of 5D digital $\mathrm{CH}$ Models (3D geometry, plus time evolution, plus the LoD) under a cost-effective way for everybody (all $\mathrm{CH}$ community players - archaeologists, architects, historians, curators, visitors, creative industries) from everywhere (remote access across different platforms, network channels, and user's preferences).

Spatio-temporal dependency means that a 3D model at a current time instance is generated taking into account information of the same object at previous time periods (temporal dependency) and common surface properties (spatial dependency). Predictive assessment indicates to $\mathrm{CH}$ community users' selection of regions of interest to be reconstructed differently on fusing/integrating selective $3 \mathrm{D}$ capturing methodologies with respect to surface features (material and geometric properties) and users' needs.

Semantic enrichment allows the 5D models to be accompanied with high level textual/visual metadata (semantics) so as to (i) assist cultural objects documentation through linking of similar $\mathrm{CH}$ assets and (ii) to perform 3D digitalization process under a cost effective way since similar surfaces' templates from one object can be exploited to reconstruct other surfaces (joint reconstruction).

The proposed framework accelerates the degree of automation for $\mathrm{CH}$ object capturing. This is achieved via the use of an automatic selective capturing framework that identifies the most suitable capturing procedure for each object surface (according to its material and constructional details-smooth/rough surface), LoD needed and time required, as well as based on advanced computer vision algorithms (SLAM) to allow real-time 3D measurements and adjustments of the initial selective estimates to real-life conditions. 
The rest of this paper is organized as follows: Section 2 presents previous works. Section 3 proposes the 5D modelling approach. Change motion maps are depicted in Section 4. Section 5 describes the database system and the visualization framework along with the CityGML model. An additional Virtual Reality viewer is described in Section 6. Finally, conclusions are drawn in Section 7.

\section{PREVIOUS WORKS}

Nowadays, several methods exist in 3D modelling: i) image based methods that exploit photogrammetric aspects in creating high fidelity 3D maps (Remondino and Zhang, 2006; Hullo et al., 2009; Haala and Rothermel, 2012), ii) photometric stereo that exploits light reflection properties for 3D modelling (Argyriou et al., 2014), iii) real-time depth sensors, such as Kinect, to create cost-effective but of low fidelity RGBD images (Izadi, 2011), iv) structured light technologies with the capability of simultaneously capture 3D geometry and texture (Soile et al., 2013; Orghidan, 2014) and v) laser scanning for large scale automated 3D reconstruction (Valanis et al., 2009; Hai, 2013). However, each of the aforementioned methods present advantages/disadvantages making high fidelity 3D modelling of $\mathrm{CH}$ assets a complex decision making process.

Automate photogrammetric matching techniques presents the advantage of creating high fidelity $3 \mathrm{D}$ point clouds, but the respective accuracy significantly falls in cases of uniform texture images, which is a usual case as regards cultural assets. Photometric stereo can be applied either for improving the results of image based matching or for reconstructing transparent/specular surfaces, where conventional methods fail. Real time depth sensors, such as Kinect, present the advantage of providing a cost-effective $3 \mathrm{D}$ modelling.

However, the respective 3D meshes are of low resolution and therefore not suitable for many $\mathrm{CH}$ application scenarios (e.g., reconstruction, monitoring). Structured light $3 \mathrm{D}$ methods are suitable for high accuracy and LoD modelling, but present difficulties in scanning large dimensions archaeological sites. Finally, 3D laser scanning presents the advantage of automation, but it fails in capturing textured point clouds and the cost of $3 \mathrm{D}$ modelling is high due to use of expensive terrestrial laser scanners.

Despite the existence of several 3D capturing methods, 3D modelling is currently performed under an isolated and fragmented solution without taking into consideration the advantages/disadvantages of each technology as well as the requirements of the respective application domain (e.g., uniform regions, transparent surfaces, low vs high resolution 3D modelling, large vs small dimension modelling).

In particular, each research institution and/or enterprise adopts a specific 3D modelling policy at which they have specialized at. In this context, integration means that the advantages of one method are used to adjust the operation (or parameters) of other $3 \mathrm{D}$ modelling approaches, improving both the resolution accuracy and the respective cost. For example, let us suppose that we have an initial 3D model obtained from low fidelity depth sensors; then, by applying $3 \mathrm{D}$ processing algorithms we can determine the regions that need more accurate 3D reconstruction (e.g., regions of complex geometric features), compared with the surfaces that need low resolution accuracy (e.g., regions of simple geometry).

Another important limitation of the current 3D modelling methods is the lack of automation in $3 \mathrm{D}$ modelling decision making. Automation means to determine a list of parameters that defines, i) the regions (part of an object or a surface) of 3D modelling, ii) the levels of details (e.g., the respective resolution), iii) the respective $3 \mathrm{D}$ technologies (or a

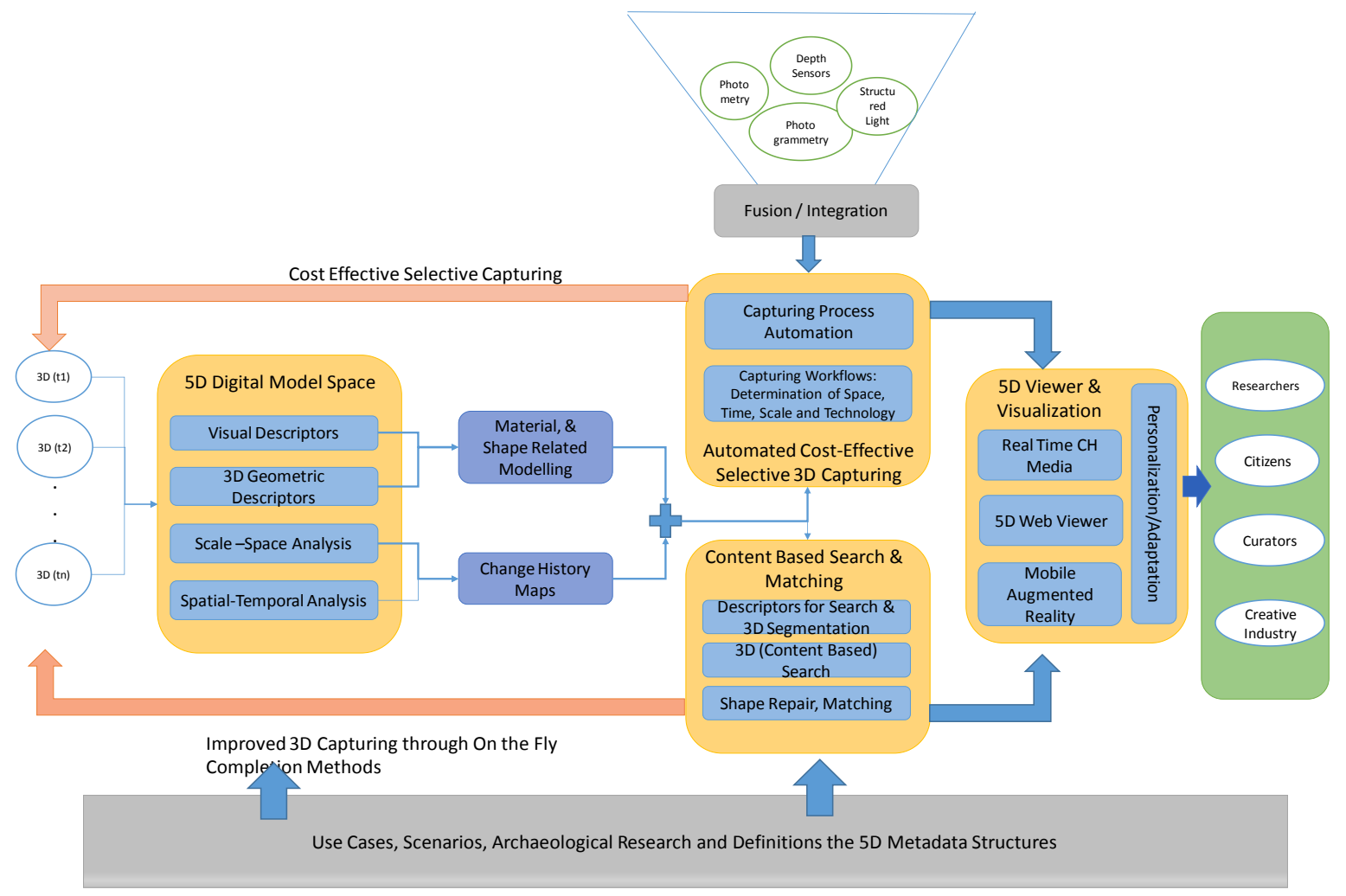

Figure 1. The overall research approach 
combination of them) and v) the time instance at which 3D modelling is performed. This quartet (region, levels of details, time, technology) determines the main parameter that automates decision making in 3D modelling. Then, through the aid of robotic methods, such as UAVs, we can automate 3D modelling processing, and therefore reduce the cost of $3 \mathrm{D}$ reconstruction.

\section{FIVE DIMENSIONAL (5D) MODELLING}

An innovative 5D modelling framework (3D geometry plus time plus different LoD) is proposed for improving the automation and cost-effectiveness of $3 \mathrm{D}$ capturing of cultural heritage assets, and for provisioning new personalized tools and services to different $\mathrm{CH}$ actors (researchers, curators and creative industries) in analyzing, documenting, understanding and experiencing cultural heritage. Figure 1 presents the overall concept of the proposed framework.

\subsection{Methodology}

The initial step is the definition of use cases, scenarios and the respective archaeological constraints used to model the research. The archaeological constraints includes the definition of the historical framework of the research, the objects to be captured, visualized and monitored, the definition of additional metadata that describes the whole research process, such as archaeological description, material properties, conservation and reconstruction features, environmental and additional contextual factors. The output of this first step is of crucial importance for the success of the proposed framework.
The core of the framework is the 5D digital model that includes three main aspects of information; the 3D geometric properties of the captured cultural heritage object, the respective time information and the levels of details representation. The generation of 5D digital models involves four steps. The first step deals with the visual representation of a $3 \mathrm{D} \mathrm{CH}$ asset. Visual representation refers to colour/texture properties.

The second step is the categorization of the shape - related objects' properties. This is performed by extracting 3D features directly applicable onto 3D object mesh. This representation is required for spatial-temporal analysis of the $3 \mathrm{D}$ meshes in order to create the predictive $3 \mathrm{D}$ modelling scheme.

The third step deals with the scale (LoD) space analysis algorithms to allow a scalable representation of the captured 3D $\mathrm{CH}$ asset (or parts of it) under different levels of details, organizing 3D meshes into a multi-level pyramid framework. Finally, the last step includes the spatial-temporal analysis where temporal change history maps are created. These history maps allow for a cost-effective capturing process since at forthcoming time intervals only regions of changes are detected and being captured using high resolution accuracy.

Using the proposed selective (predictive) 3D modelling scheme only regions that undergo a significant spatial-temporal change are $3 \mathrm{D}$ modelled at the next time instance. On the contrary, regions of insignificant change remain intact. For this reason, a spatial temporal analysis is initially applied on to the 3D models
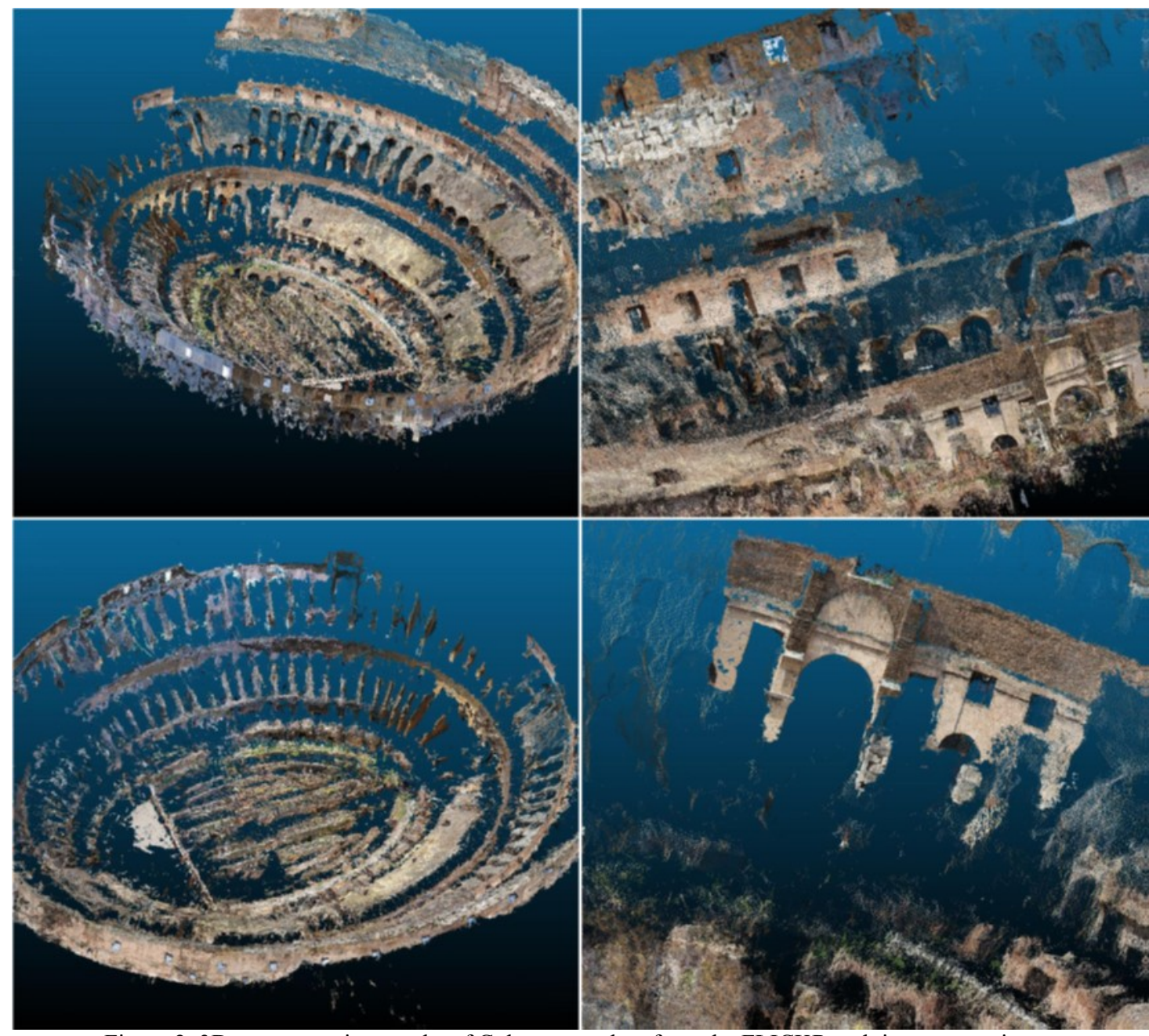

Figure 2.3D reconstruction results of Colosseum taken from the FLICKR web image repository. 
to indicate the regions of changes. The change history maps provide useful information that automates the 3D capturing process, introduce a cost-effective $3 \mathrm{D}$ acquisition framework.

The outcome of the spatial-temporal analysis is exploited for determining the most appropriate photogrammetric or other method for the next $3 \mathrm{D}$ modelling. Therefore, regions of insignificant changes can be captured with less precise 3D techniques, avoiding computational complexity. On the other hand, regions of significant changes are 3D scanned with precise methods, since no significant information is available from previous 3D modelling processes.

The results may be visualized into interoperable viewers, operating based on the CityGML framework. CityGML allows the description of additional semantic metadata information, such as the rights of the land resources. Visualization aspects are also supported to allow easy manipulation, interaction and representation of the models and the respective semantic information.

\subsection{D Modelling from Web Data}

A fast and cost effective low resolution 3D modelling of a monument may be achieved using automated methods and data taken from the web. One of the main characteristics of Internet era is the free and online availability of extremely large collections of images located on distributed and heterogeneous platforms over the web. In contrast to the approaches where 3D data acquisition is accomplished in a very constrained environment, using specialized equipment, Web-based collections ("wild image collections") may be exploited for cultural heritage e-documentation.

This means that user generated content can be exploited to find images taken from simple users than a $3 \mathrm{D}$ reconstruction to assess a monument through time. This decreases a lot the cost of reconstruction. Instead of taking data in the field, we can exploit data from simple users to find out regions of interest that may be useful for the assessment and documentation process. Content-based retrieval mechanisms may be activated to improve the analysis and the reconstruction from data taken from the web.

However, the main difficulty of using "wild image collections" is that the Internet stored image content is unstructured, requiring new tools in the area of content-based filtering. The implementation of a precise 3D/4D reconstruction of an object from unstructured internet image collections, being captured for personal use instead of reconstruction purposes, is that there are several outliers in the set of retrieved data deteriorating performance and increasing computational cost.

While there exist 3D reconstruction algorithms, such as the structure from motion which present robustness against noisy data, their computational complexity significantly increases with respect to the number of input data. This makes direct implementation of such methods, for large image volumes, practically impossible.

Figure 2 shows some 3D reconstruction results from Colosseum stadium in Rome. The images were taken for touristic purposes from simple users and these photos were uploaded on the Flickr image repository. The results were compared with 3D reconstruction methods taken from semi-automatic methods involving manual processes. In particular, Figure 3 a shows the reconstruction of a monument in Germany (Porta Nigra) using images from the web; Figure $3 b$ shows precise results using semi-automatic methods.

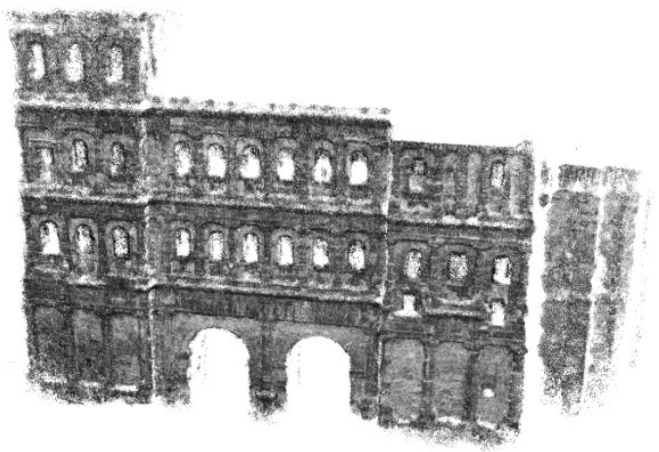

(a)

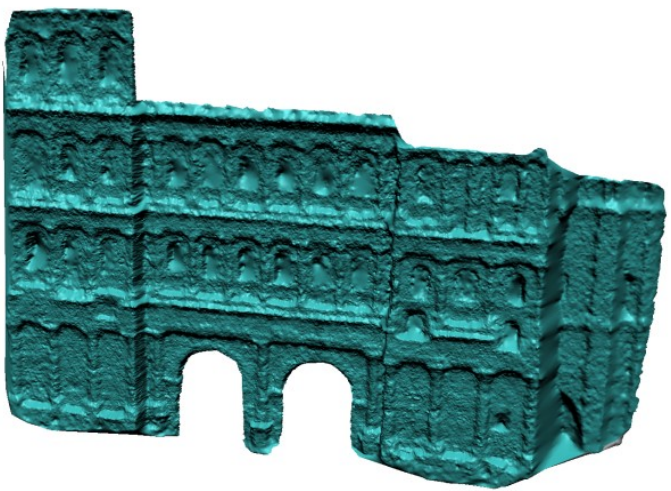

(b)

Figure 3. (a) 3D reconstruction results of Porta Nigra taken from the web image repositories; (b) 3D reconstruction results of Porta Nigra using semiautomatic methods.

\section{CHANGE HISTORY MAPS}

Change history maps (Figure 4) detect regions of interest in the $3 \mathrm{D}$ space by combining multiple instances of a $3 \mathrm{D}$ model. This way, we can support the selective partial acquisition of a cultural heritage resource, which significantly accelerates the effort of 3D modelling especially in cases of time varying assets. The change history map determines the regions that need to be reconstructed more precisely than others due to temporal changes.

\subsection{Geometric History Changes}

The first approach for creating change history maps is through the geometric differences of the 3D models. A simple way is to apply a point by point difference. However, to address noise effects, we initially smooth the 3D models by the application of a Gaussian pyramid filter. Then, change history maps are created directly from the filtered $3 \mathrm{D}$ models. Another important aspect is the appropriate alignment of the 3D models between two different time instances. For this reason, the ICP algorithm is applied between the two examined $3 \mathrm{D}$ models in order to align the point clouds of the two time instances.

Figure 4 presents the concept of the geometric history maps. The spatial regions where a significant change is encountered are depicted as a rectangle. The three different colours indicate the resolution accuracy of the 3D modelling. 
t1
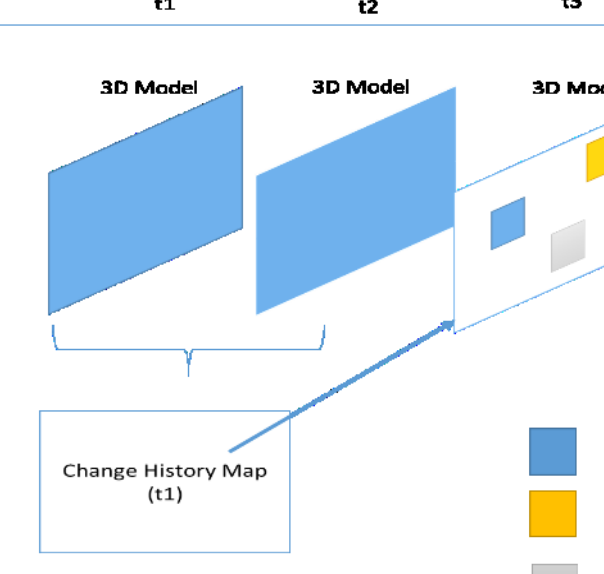

Low Resolution

Medlum Resolution

High Resolution

No Capturing

Figure 4. The research approach adopted as regards spatialtemporal analysis

\subsection{Semantic History Changes}

Another factor for determining the change history maps is through the metadata information embedded on the $3 \mathrm{D}$ models. Each part of the cultural heritage resource is represented by a set of metadata information. These metadata may refer, for example, to architectural, topographical, imagery, archaeological and other information. In case that the geometric properties of a part of the $\mathrm{CH}$ asset have not been changed but the semantic information connected with a part of the resource has, a change history map is also created. However, this does not imply a new 3D reconstruction process is required, but instead specifies that the semantic metadata information has been updated.

The aforementioned approach is elaborated with an example. It is assumed that a spatial region in the cultural heritage resource has been initially modelled with a high accuracy 3D capturing process and that this region goes through a spatial transformation in the following time period. A low resolution fully automated $3 \mathrm{D}$ modelling procedure takes place to identify the changes of the initial 3D model. In the case that changes are identified, the low accuracy model of the second time period is improved locally by enhancing the low resolution automatically captured 3D data to high accuracy representations.

\section{DATABASE STORAGE AND VISUALIZATION}

Using the proposed 5D modelling approach, heterogeneous data associated with different time and LoD information will be collected (analysed and semantically enriched), including multiple raster (e.g., terrestrial or aerial images) and vector files (e.g., shapefiles), as well as additional metadata information (e.g., from an archaeological cadastre database). In order to be able to perform specific queries and visualizations using this information, it was decided to first transform them to an extended-CityGML format and semantically enrich them before storing them to a database.

For this reason, all information is finally stored in a PostgreSQL/PostGIS database, which is open-source, crossplatform and suitable for handling spatial information. The storage includes both CityGML objects, representing the 3D (geometrical) information, as well as additional metadata information that are linked to the corresponding CityGML objects. To this end, tools were developed to query the database based on specific metadata (e.g. region of interest, material properties, etc.) and visualize the $3 \mathrm{D}$ models resulting from the query along with the associated semantic metadata information.

Regarding the fourth-dimension (time), monuments (or parts of a monument) that undergo changes between two consecutive time instants will be associated with two different IDs, while a single ID will be used if no change occurs. The additional time (START/END date) that will be added as an external reference in the database will be used to encode changes in the time dimension. Future extensions making use of GML temporal objects could be examined, however, their support is more suitable for future CityGML versions.

For visualization of CityGML models that are produced by database queries, the viewers should support the following features: a) Controls allowing to interactively visualize different time instants and levels of details, b) Option to display (or highlight) the changes between two models (e.g., from two different time instants), c) Display of additional associated metadata.

Regarding the input data formats a number of visualization approaches are available. The first approach is direct visualization of the geometry of CityGML. Some free userfriendly interfaces exist for visualizing CityGML models, including the Aristoteles3D viewer (http://www.geokiosk.net/explore-3dgeo/ Institute for Cartography and Geoinformation, University of Bonn) and others (van Oosterom et al., 2008; Iñaki et al., 2002), but extending of these viewers to display metadata information and support time/scale is not an easy task.

A second approach is to first transform CityGML to a more efficient file format, for which viewers already exist and/or which can be displayed more easily. Most relevant open formats which are interoperable with CityGML and suitable for such an approach are a) KML/COLLADA to display 3D models in Google Earth and b) X3D (the successor of VRML) to display models in any modern browser. Both KML and X3D have support for time data/time stamps as well as for data animations. Therefore, suitable exporters of data in these formats can be developed, so that changes in the time and scale dimension can be properly visualized.

In the first option, the exporter generated a single KML file that can be then loaded to Google Earth. The user is able to playback an animation that shows the evolution of the $3 \mathrm{D}$ models over time and/or navigate in 3-D space to view the data. In addition, different scales of the cultural objects/archaeological site are displayed interactively, depending on the distance of the observer from the objects/site (i.e. when the camera is far from an object, LOD1 is loaded, if it approaches more, LOD2 is loaded, and if it is very close, LOD3 is loaded). This is achieved using the Level of Detail feature, which is supported by KML. Associated metadata information corresponding to each 3D models can also be visualized in a popup window, using the KML BalloonElement.

Another option for achieving efficient visualization is by converting the CityGML data to X3D. X3D (http://www.web3d.org/standards) is a royalty-free open standard file format to represent and communicate $3 \mathrm{D}$ scenes and objects using XML. Then, a WebGL interface can be used 
for visualization of CityGML into any WebGL enabled browser (e.g. chrome, firefox, etc.) without need for any plugin.

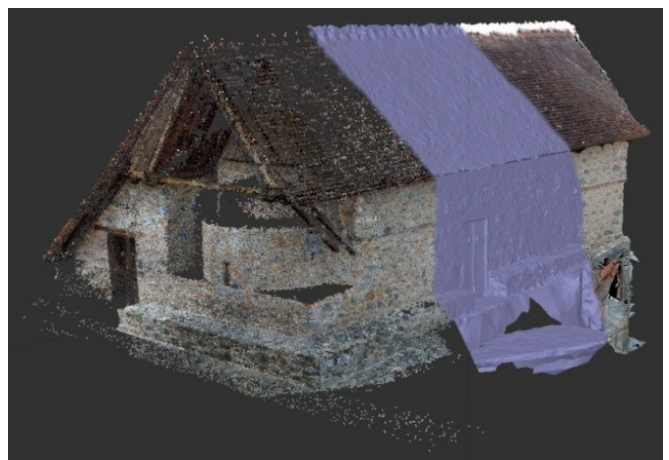

Figure 5. 3D reconstruction of Archangelos Michael church using an image-based technique. From left to right: point cloud, 3D mesh, textured 3D mesh.

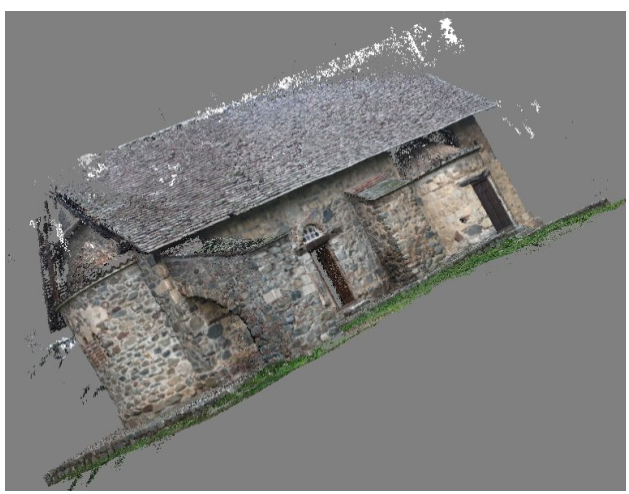

Figure 6: A 3D reconstruction model of The Panayia Phorviotissa or Asinou church.

\section{VIRTUAL REALITY VIEWER}

The Virtual Reality (VR) viewer (Doulamis et al, 2015) is an attempt to create an end-user software which manages the presentation of the various data-sets (imagery, 3D scans, 3D models, etc.). As a case study, the 3D model of Archangelos Michael church with different information levels is presented (Figure 5). The church is located on the mountain of Troodos, Cyprus and it is included in UNESCO World Heritage Sites along with other 9 painted Byzantine Churches of Cyprus (http://www.byzantinecyprus.com).

Another example is the reconstruction of Panayia Phorviotissa or Asinou church, located also on the mountain of Troodos in Cyprus (http://www.byzantinecyprus.com). It was initially erected around $1100 \mathrm{AD}$ and suffered serious damages since. then that lead to partial re-buildings and wall redecorations of the monument. In order to fully reconstruct the monument in 3D (Figure 6) with the use of structure from motion (SfM) and multi-view stereo (MVS) techniques, various images of the exterior of the church were taken using a Canon 500D DSLR CMOS camera. For a full 3D reconstruction, images from various aspects (different orientations and scales) should be acquired. SfM techniques do not require strict camera geometries between the stereo-pairs, as they are robust enough to work also with random and unordered image sequences.

In Figure 7 the main idea of the proposed 5D modelling is presented. The idea is to have a high resolution detailed model (Figure 7-left) at a first time instance. Then, a low resolution model (Figure 7-right) will be created at a next time instance, using fully automated image-based techniques. Though the comparison/matching of specific parts of the 3D models of the two time instances, regions of the monument which remain unchanged are detected, we do not need to reconstruct from scratch the accurate $3 \mathrm{D}$ model of the whole monument at the second time instance. Instead, we can use these parts of the high resolution model and move them to the low resolution model.

\section{CONCLUSIONS}

In order to accomplish an efficient, cost-effective cultural heritage asset representation for ever, everybody and from everywhere, a 5D multimedia digital model (namely the 5D Digital Cultural Heritage Model - 5D-DCHM) was proposed. The development of such a model, which shows the development of a 3D model of a part of a Cultural Heritage resource through time, may have multiple uses and applications.

The application of the proposed selective (predictive) 4D modelling framework may improve automation and costeffectiveness of 3D capturing of cultural heritage resources, since only the spatial 3D differences are modelled at the forthcoming time instances. So, the cost of the creation of accurate 3D models in various time instances is eliminated, while regions of no significant spatial-temporal alterations remain intact.

The new model is capable of detecting material degradation factors through time, the effect of environmental phenomena on the objects (humidity, erosion), structural problems and defects (cracks, sprawls) and potential failures caused by previous erroneous restoration actions.

Conservators can assess the impact of previous methods applied and test more suitable solutions to retain the objects' details. Chemical engineers can identify potential problems of objects'
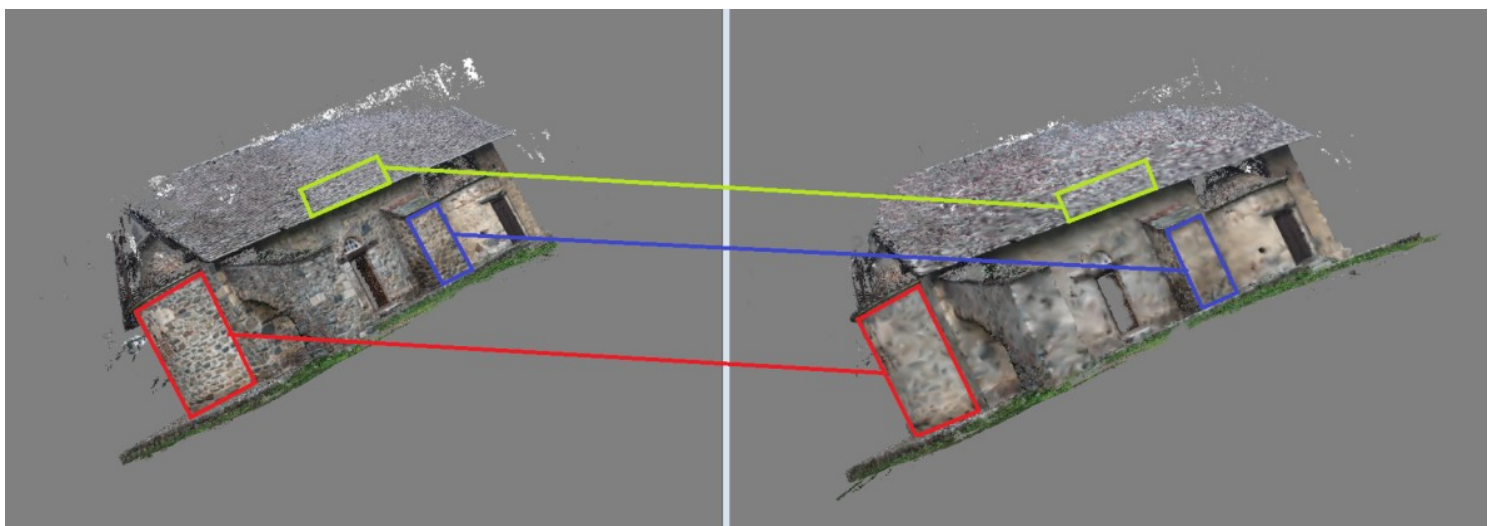

Figure 7. The implementation of the proposed modelling approach on the Asinou church. 
material and intervene to decay the degradation process. Archaeologists are aware of the impact of different excavation methods on the object structure and take actions according to the type of material they expect regarding excavation case.

Furthermore, the digital counterpart of an object will allow $\mathrm{CH}$ researchers to make precise replicas that resemble not only the 3D geometric structure but also material used. They can also use model that simulate environmental phenomena. This way, the digital model can retain objects' details even if an unexpected disaster can occur like a high intense earthquake, loot, and/or mankind destruction (war, fire).

An open source PostgreSQL database was used to manage 3D data and their semantics. The proposed representation a) stores efficiently the 3D data in CityGML format as well as their associated semantic metadata information and b) supports the interactive visualization of the 5D-DCHM, including time/scale animations and metadata display.

\section{ACKNOWLEDGEMENTS}

This research was conducted in the framework of the research project "5 Dimensional Multi-Purpose Land Information System" (5DMuPLIS). For the Greek side 5DMuPLIS project is co-funded by the EU (European Regional Development Fund/ERDF) and the General Secretariat for Research and Technology (GSRT) under the framework of the Operational Programme "Competitiveness and Entrepreneurship", "GreeceIsrael Bilateral R\&T Cooperation 2013-2015".

It also supported by the following two EU projects:

1) FP7-PEOPLE ITN2013 ITN-DCH project "Initial Training Network for Digital Cultural Heritage" This project has received funding from the European Union's Seventh Framework Programme for research, technological development and demonstration under grant agreement no. 608013

2) FP7-IAPP2012 4D-CH-WORLD project "4D Cultural Heritage World" This project has received funding from the European Union's Seventh Framework Programme for research, technological development and demonstration under grant agreement no. 324523

\section{REFERENCES}

Argyriou, V, Zafeiriou, S., Petrou, M, 2014. Optimal illumination directions for faces and rough surfaces for single and multiple light imaging using class-specific prior knowledge. Computer Vision and Image Understanding.

Bjerke B. and Karlsson, M., 2013. Social entrepreneurship: To act as if and make a difference. Edward Elgar Publishing.

Doulamis, A., Doulamis, N., Makantasis K., Klein M, A 2015. 4D Virtual/Augmented Reality Viewer exploiting Unstructured Web-based Image Data. International Conference on Computer Vision Theory and Application, Berlin, Germany.

Ene, D. and Radvan, R., 2011. Digital model for cultural heritage conservation status evaluation, In: 4th International Conference on Developments in eSystems Engineering, DeSE.

Gomes, L., Pereira Bellon O.R., Silva, L, 2014. 3D reconstruction methods for digital preservation of cultural heritage: A survey. Pattern Recognition Letters.
Haala, N. and Rothermel, M., 2012. Dense multiple stereo matching of highly overlapping uav imagery. ISPRS International Archives of the Photogrammetry, Remote Sensing and Spatial Information Sciences XXXIX-B1, pp. 387-392.

Hai H., Brenner, C., Sester, M., 2013. A generative statistical approach to automatic 3D building roof reconstruction from laser scanning data. ISPRS Journal of Photogrammetry and Remote Sensing, Vol. 79, pp. 29-43.

Hullo, J. F., Grussenmeyer, P., Fares, S., 2009. Photogrammetry and Dense Stereo Matching Approach Applied to the Documentation of the Cultural Heritage Site of Kilwa (Saudi Arabia). In: $22^{\text {th }}$ CIPA Symposium. Kyoto, Japan.

Iñaki, I., Izkara, J.L., Delgado, F. J., 2002. From point cloud to web 3D through CityGML. In: 18th international conference on Virtual systems and multimedia (VSMM).

Izadi, S., Kim, D., Hilliges, O., Molyneaux, D., Newcombe, R., Kohli, P, Shotton, J., Hodges, S., Freeman, D., Davison, A., Fitzgibbon, A., 2011. KinectFusion: Real-time 3D Reconstruction and Interaction Using a Moving Depth Camera. ACM UIST'11, Santa Barbara, CA, USA.

Kitamura, K., 2013. Common software for digital exhibition of Japanese cultural heritage in literature. In: International Conference on Culture and Computing, Culture and Computing, 6680347, pp. 137-138.

Makantasis, K., Doulamis, A., Doulamis, N., Ioannides, M. 2014. In the wild image retrieval and clustering for 3D cultural heritage landmarks reconstruction. Multimedia Tools and Applications, Article in Press.

Orghidan, R., Salvi, J., Gordan, M., Florea, C., Batlle, J., 2014. Structured light self-calibration with vanishing points. Machine Vision and Applications, Vol. 25, No. 2, pp 489-500.

van Oosterom, P.. and Stoter, J., 2010. 5D Data Modelling: Full Integration of $2 \mathrm{D} / 3 \mathrm{D}$ Space Time and Scale Dimensions, S.I. Fabrikant et al. (Eds.): GIScience 2010, LNCS 6292, pp. 310324, Springer-Verlag Berlin Heidelberg.

van Oosterom, P. and Stoter, J., 2012. Principles of 5D modelling, GIS technology. Geospatial World Weekly, Available http:// geospatialworld.net /Paper/ Technology/ArticleView.aspx?aid=30344

van Oosterom, P., Zlatanova, S., Penninga, F., 2008. Advances in $3 \mathrm{D}$ geoinformation systems. Springer.

Remondino, F. and Zhang, L., 2006. Surface reconstruction algorithms for detailed close range object modelling. In: International Archives of Photogrammetry, Remote Sensing and Spatial Information Sciences, Vol. XXXVI, pp. 117-123.

Soile, S., Adam, K., Ioannidis, C., Georgopoulos, A., 2013. Accurate 3D textured models of vessels for the improvement of the educational tools of a museum. International Archives of the Photogrammetry, Remote Sensing and Spatial Information Sciences, Volume XL-5/W1, pp. 211-217.

Valanis, A., Tapinaki, S., Georgopoulos, A., Ioannidis C., 2009. High resolution textured models for engineering applications. 22nd CIPA Symposium, Kyoto, Japan. 